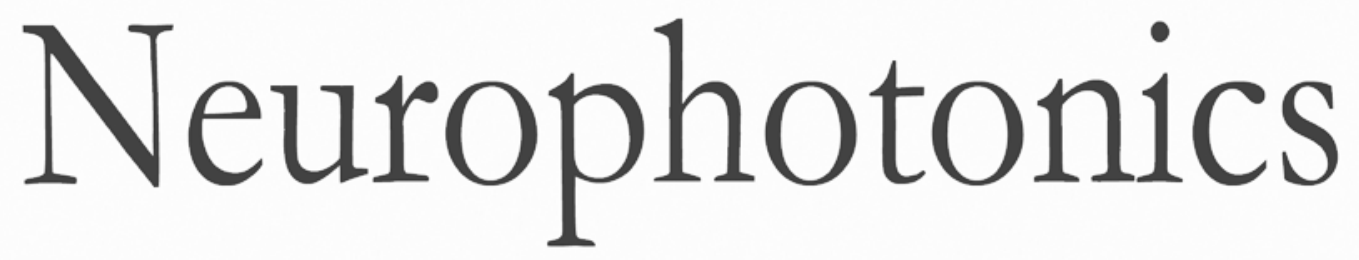

Neurophotonics.SPIEDigitalLibrary.org

\title{
Review on photoacoustic imaging of the brain using nanoprobes
}

Depeng Wang

Yun $\mathrm{Wu}$

Jun Xia

\section{SPIE.}




\title{
Review on photoacoustic imaging of the brain using nanoprobes
}

\author{
Depeng Wang, Yun Wu, and Jun Xia* \\ State University of New York, University at Buffalo, Department of Biomedical Engineering, 208 Bonner Hall, Buffalo, \\ New York 14260, United States
}

\begin{abstract}
Photoacoustic (PA) tomography (PAT) is a hybrid imaging modality that integrates rich optical contrasts with a high-ultrasonic spatial resolution in deep tissue. Among various imaging applications, PA neuroimaging is becoming increasingly important as it nicely complements the limitations of conventional neuroimaging modalities, such as the low-temporal resolution in magnetic resonance imaging and the low depth-to-resolution ratio in optical microscopy/tomography. In addition, the intrinsic hemoglobin contrast PA neuroimaging has also been greatly improved by recent developments in nanoparticles (NPs). For instance, nearinfrared absorbing NPs greatly enhanced the vascular contrast in deep-brain PAT; tumor-targeting NPs allowed highly sensitive and highly specific delineation of brain tumors; and multifunctional NPs enabled comprehensive examination of the brain through multimodal imaging. We aim to give an overview of NPs used in PA neuroimaging. Classifications of various NPs used in PAT will be introduced at the beginning, followed by an overview of PA neuroimaging systems, and finally we will discuss major applications of NPs in PA neuroimaging and highlight representative studies. ๑ 2016 Society of Photo-Optical Instrumentation Engineers (SPIE) [DOI: 10.1117/1.NPh.3.1.010901]
\end{abstract}

Keywords: photoacoustic tomography; neuroimaging; nanoparticle.

Paper 15038VRR received Aug. 12, 2015; accepted for publication Nov. 24, 2015; published online Jan. 4, 2016.

\section{Introduction}

Photoacoustic (PA) tomography (PAT) ${ }^{1,2}$ is an emerging imaging modality that provides noninvasive high-resolution (20 to $100 \mu \mathrm{m}$ ) images of the entire brain in a preclinical setting at near real-time (temporal resolution $<100 \mu \mathrm{s}$ ). PAT forms images by detecting the acoustic pressure induced by thermoelastic expansion following the absorption of optical energy, e.g., from laser light irradiation. Hence, the image contrast of PAT is primarily determined by the light absorption properties of the tissue, and the imaging frame rate is only limited by the laser's pulse repetition rate $(10 \mathrm{~Hz}$ to $5 \mathrm{kHz})$ and the speed of sound in tissue (typically $1.5 \mathrm{~mm} / \mu \mathrm{s}$ ). In brain tissues, the dominant endogenous optical absorber is hemoglobin, which enables label-free vascular imaging. Multispectrum PAT imaging allows the quantification of oxy- and deoxyhemoglobin $\left(\mathrm{HbO}_{2}\right.$ and $\left.\mathrm{Hb}\right)$ concentrations, which reveals oxygen saturation, an important physiological parameter that is difficult to measure with many other neuroimaging modalities. The broad choice of optical contrast agents, such as nanoparticles (NPs) ${ }^{3}$ chemical dyes, ${ }^{4}$ and fluorescence proteins, ${ }^{5,6}$ further enhances the imaging capability of PAT, allowing for molecular brain imaging.

Compared with conventional contrast agents, NPs possess several unique advantages for PAT: (1) NPs' optical absorption spectrum can be fine-tuned to meet specific imaging applications; (2) several targeting molecules can be integrated into one NP structure to enhance PAT specificity on identifying brain tumors; (3) some NPs' rigid metallic structures are not susceptible to photobleaching, allowing for prolonged brain imaging; and (4) NPs' large surface area or interior volume allows easy loading of imaging agents and drugs, enabling multimodal

*Address all correspondences to: Jun Xia, E-mail: junxia@buffalo.edu brain imaging and therapy. With these unique features, NPs are increasingly used in PA neuroimaging. This review aims to give an overview of developments in the field. Section 2 talks about the classification of NPs used in brain PAT. Section 3 reviews the five implementations of PA neuroimaging systems. Then, we discuss three major applications of NPs in PA neuroimaging and highlight representative studies. Finally, we conclude the review and discuss future directions. It should be noted that this review focuses only on NP-enabled PA neuroimaging. General reviews on PA neuroimaging or NPs used in PAT can be found in Refs. 7-9 and 10-12, respectively.

\section{Classification of Nanoparticles Used in Photoacoustic Brain Imaging}

NPs, in general, refer to particles varying in diameter from 1 nanometer to several hundred nanometers. For biomedical imaging, most NPs have a size less than $100 \mathrm{~nm}$ to avoid rapid clearance from systemic circulation. ${ }^{13}$ At this dimension, particles exhibit unique optical properties from quantum effects and thus have a wide variety of applications in electronics, optics, and biomedicine. ${ }^{14,15}$ In this section, we give an overview of NPs that have been used in PAT in general and highlight the ones for PA neuroimaging. Our discussion will be based on the composition of NPs: organic NPs, inorganic NPs, and hybrid NPs. ${ }^{10}$

Organic NPs are formed with organic molecules that are bound together by chemical binding or self-assembly. Their good biocompatibility makes them appealing contrasts for in vivo imaging. Notable organic NPs used in PAT include porphysomes, ${ }^{16}$ perfluorocarbon-based (PFC) nanodroplets, ${ }^{17,18}$ polymer NPs, ${ }^{19-21}$ hydrogel NPs ${ }^{22}$ and perylene-3,4,9,10-tetracarboxylic diimide (PDI) NPs. ${ }^{23}$ Porphysomes have a liposome-like structure and are one of the first organic NPs designed to have strong near-infrared (NIR) absorption. They 
also exhibited excellent biocompatibility: even after a high-intravenous injection dose ( $1 \mathrm{~g} / \mathrm{kg}$ mouse weight), mice still behaved normally, and a histopathological examination indicated good liver, spleen, and kidney functions. PFC nanodroplets were originally developed for ultrasound imaging. PA contrast can be introduced by loading nanodroplets with light absorbing materials. For instance, in indocyanine-green-loaded PFC nanodroplets, the PA contrast originated from indocyanine green (ICG), which is a food and drug administration approved contrast that absorbs around $800 \mathrm{~nm} .{ }^{17}$ The intense optical absorption also makes the nanodroplets vaporize, which subsequently enhances the ultrasound contrast. ${ }^{24-27}$ Among various polymer NPs, semiconducting polymers nanoparticles such as those made from poly (cyclopentadithiophene-alt-benzothiadiazole) are distinguished by their strong per-mass PA contrast and narrow absorption spectrum. In addition, they are resistant to photodegradation and oxidation, enabling in vivo imaging of reactive oxygen species, which is an important chemical mediator for many diseases. ${ }^{28}$ Hydrogel NPs are made of polyacrylamide (PAA) matrices with covalently linked Coomassie blue (CB) dye molecules. ${ }^{22}$ They are biocompatible, chemically inert, and nontoxic, and are the first type of organic NPs used in PA neuroimaging. However, their absorption in the visible region precludes in vivo deep-brain imaging. PDI is a class of small molecules that have exceptional chemical and photochemical stabilities and are extremely inexpensive. Their small molecule size and strong NIR absorption allow the imaging of deep brain tumors in live mice. Compared with inorganic NPs, organic PAT NPs have a relatively short development history and thus have only been used in limited PA neuroimaging studies.

Inorganic NPs used in PA include metallic NPs, ${ }^{29}$ quantum dots (QDs), ${ }^{30,31}$ iron oxide NPs, ${ }^{32,33}$ carbon nanotubes, ${ }^{34-37}$ and upconversion NPs. ${ }^{38}$ Gold NPs are the most popular group of metallic NPs used in PAT and PA neuroimaging, owing to their stable structure and strong extinction peak in the visible and NIR regions. ${ }^{39}$ By manipulating the size, shape, and internal structure, a variety of gold NPs, such as gold nanocages,${ }^{39}$ gold nanoshells, ${ }^{40}$ and nanospheres, ${ }^{41}$ have been developed with different optical and loading capabilities. As will be discussed in the 3, gold NPs have been used in PA neuroimaging to enhance the vascular and tumor contrast. QDs are nanocrystals with sizes ranging from 2 to $10 \mathrm{~nm}^{42}$ Their small size enhances transmigration across the blood brain barrier (BBB), ${ }^{43}$ which protects the brain from harmful substances but also prevents the effective delivery of therapeutic agents. QDs have excellent photostability, a large absorption cross section, and a high-quantum yield. They have been used in multimodal fluorescence, PA, and photothermal (PT) imaging. ${ }^{44}$ Iron oxide NPs were originally developed as magnetic resonance imaging (MRI) contrast agents. ${ }^{45}$ Bare iron oxide NPs have poor absorption in NIR and are thus not suitable for deep brain imaging. Silica coating has been proposed to enhance their NIR absorption. ${ }^{46}$ Carbon nanotubes are characterized by their small diameter ( 1 to $2 \mathrm{~nm}$ ) and high aspect ratio (50 to $300 \mathrm{~nm}$ in length), which mitigates their clearance by the reticuloendothelial system. ${ }^{34}$ Similar to other NPs, carbon nanotubes have been used to enhance the tumor contrast in PAT. Upconversion NPs convert NIR absorption into emission in the visible region. However, their recent application in PAT was based on the luminescence quenching effect. ${ }^{38}$ In general, while they are less biocompatible, inorganic NPs have better optical tunability and are more stable than organic NPs. However, some inorganic NPs, such as iron oxide, have already been used to image human brains, and thus hold great promise for clinical PA neuroimaging. ${ }^{47}$

There is an another class of NPs called hybrid NPs, consisting of both inorganic and organic NP components. For instance, a gold-nanorod-encapsulated PFC nanodroplet contains inorganic gold nanorods for PAT and organic PFC for ultrasound imaging. ${ }^{48}$ The gold nanorods have better photostability than ICG, which was used in Ref. 17. While hybrid NPs holds the benefits of both organic and inorganic NPs, modification to the original organic/inorganic NP structure requires further investigation of its bio and photostabilities.

\section{Photoacoustic Tomography Neuroimaging Systems}

For better understanding of imaging experiments, this section gives a brief overview of PA neuroimaging systems. More comprehensive reviews of PAT systems can be found in Refs. 1, 2, 49 , and 50. There are two major implementations of PA neuroimaging systems, one is based on photoacoustic microscopy (PAM) and the other is based on photoacoustic computed tomography (PACT). PAM is designed for high-resolution (1 to $50 \mu \mathrm{m}$ ) imaging over a shallow depth, while PACT targets at deeper imaging depth with compromised spatial resolution (50 to $200 \mu \mathrm{m}$ ).

\subsection{Photoacoustic Microscopy Systems}

According to the imaging depth and the spatial resolution, PAM can be classified into two groups: optical-resolution PAM (ORPAM) and acoustic-resolution (AR-PAM) (as shown in Fig. 1). In OR-PAM, the lateral resolution is defined by the optical focus, which ranges from submicrometer to a few micrometers. Because OR-PAM relies on ballistic and/or quasiballistic photons, the imaging depth is less than one transport mean free path (TMFP, typically $<1 \mathrm{~mm}$ in the brain). This depth is sufficient to cover the mouse cortex in an open scalp experiment. AR-PAM relies on diffusive light to form an image and its lateral resolution is defined by the acoustic focus. The imaging depth of AR-PAM is mainly determined by the penetration depth of the excitation wavelength and may vary from $1 \mathrm{~mm}$ (blue to green light) to a few mm (red to NIR light). AR-PAM can image the mouse brain cortex through an intact scalp. For both OR-PAM and AR-PAM, the axial resolution is defined by the bandwidth and central frequency of the ultrasound
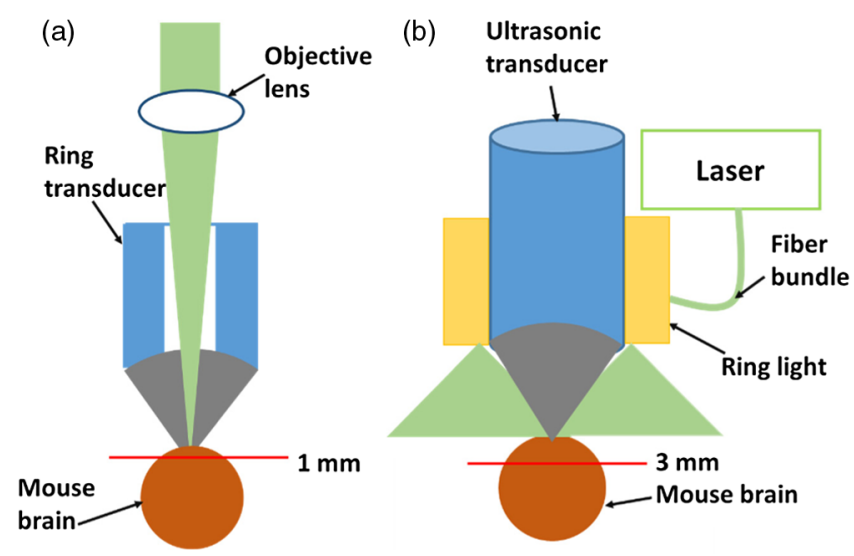

Fig. 1 Schematics of (a) optical-resolution photoacoustic microscopy and (b) acoustic-resolution photoacoustic microscopy. 
transducer. OR-PAM systems typically use higher central frequency transducers $(\sim 50 \mathrm{MHz})$, which provide better axial resolutions. Image reconstruction in PAM systems is relatively simple because the spherically focused transducer mainly captures signals along the acoustic axis. Therefore, the PAM system forms an A-line of data at each scanning position. Combining all A-lines from two-dimensional (2-D) raster scan forms a three-dimensional (3-D) image.

While PAM systems play an essential role in high-resolution functional brain imaging, ${ }^{51-53}$ they were rarely used in NP studies, probably because (1) the shallow imaging depth does not need highly NIR absorbing NPs, and (2) the raster-scan requires high pulse repetition rate $(>1 \mathrm{kHz})$ lasers, which typically lacks the wavelength tunability. Array-based PAM systems have recently been developed and may enable low-repetition rate lasers to be used in PAM. ${ }^{54-56}$

\subsection{Photoacoustic Computed Tomography Systems}

Both PACT and AR-PAM rely on diffusive photons to generate PA signals. However, transducers used in PACT are unfocused toward the imaging plane and thus require computed tomography algorithms to form an image. For systems with matching numbers of data acquisition channels and array elements, a 2-D or 3-D image can be generated from a single laser shot. ${ }^{57}$ Most PACT systems use high-power Nd:YAG lasers, operating at 10 or $20 \mathrm{~Hz}$. Wavelength tunability can be achieved through an optical parametric oscillator (OPO), a dye laser, or a Ti-sapphire laser. Based on the detection geometry, PACT can be divided into five categories: cylindrical-view PACT,${ }^{58}$ planarview PACT, ${ }^{59}$ spherical-view PACT,${ }^{60}$ linear-view PACT,${ }^{23}$ and circular-view PACT ${ }^{61}$ (Fig. 2). For the first three geometries, unfocused transducer elements are evenly, or semievenly, distributed on the scanning surface, and 3-D images can be formed with near-isotropic spatial resolution. Due to the complete 3-D coverage, exact analytical image reconstruction algorithms, such as the universal back-projection algorithm, can be used in these 3-D geometries. ${ }^{62}$ The latter two geometries have a 2-D detection coverage and exact image reconstruction is still challenging. ${ }^{63,64}$ In practice, because linear and circular arrays are cylindrically focused on the 2-D detection plane, most researchers simply use the 3-D-based reconstruction algorithm in their 2-D systems. While the reconstruction may not be quantitatively accurate, the approximation is adequate for recovering dynamic changes in the brain or for extracting strong NP absorption from the hemoglobin background. 3-D PACT systems are commonly used in volumetric neuroimaging, ${ }^{60}$ while 2 -D PACT systems are typically used for coronal- (linear-view PACT) or transverse-view (circular-view PACT) neuroimaging. It should be noted that 2-D PACT systems can also generate 3-D images by scanning vertically along the imaging plane. However, the spatial resolution along the third dimension will be poorer than their inplane resolutions. ${ }^{65}$ Advanced reconstruction algorithms or scanning geometries are needed to resolve this issue. $^{65,66}$

\section{Applications of Nanoparticles in Photoacoustic Neuroimaging}

\subsection{Contrast Enhancement}

As previously mentioned, hemoglobin is the major intrinsic PA contrast, which enables label-free functional ${ }^{67,68}$ and vascular brain imaging. ${ }^{9}$ However, in the NIR region, the optical absorption of hemoglobin is relatively weak, and contrasts from deep brain vessels are impeded by the surrounding tissue. NPs have been used to address these limitations because their absorption peaks can be systematically varied from visible to the NIR (a)

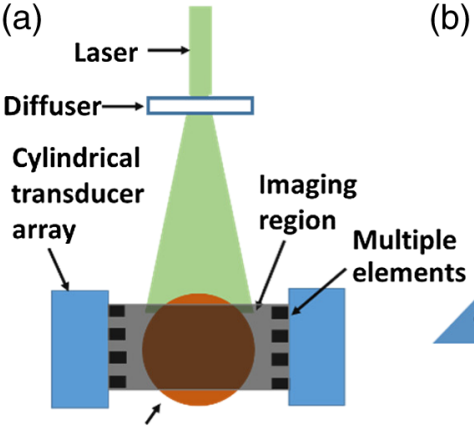

Mouse brain (b)

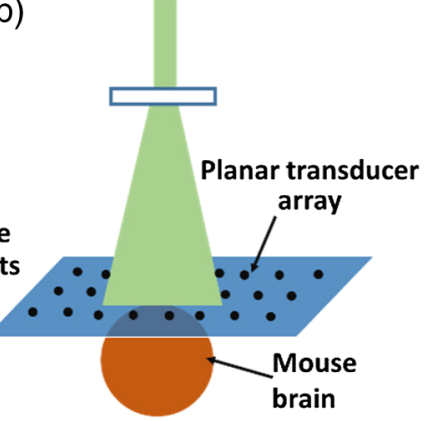

(c)

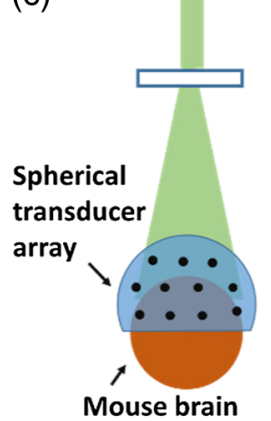

(d)

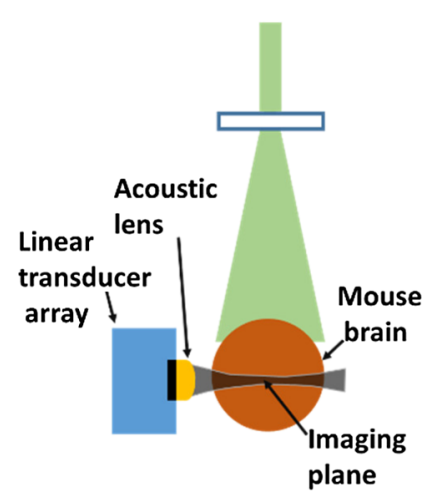

(e)

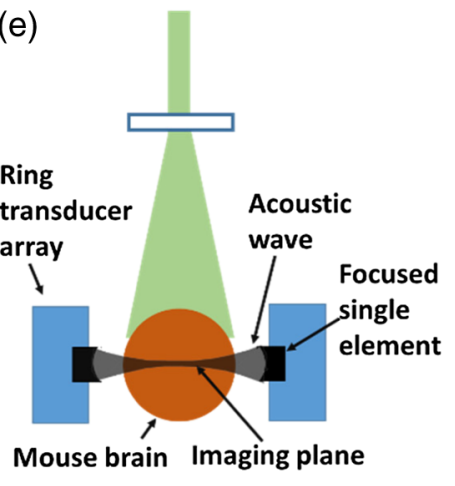

Fig. 2 Schematics of: (a) cylindrical-view photoacoustic computed tomography (PACT), (b) planar-view PACT, (c) spherical-view PACT, (d) linear-view PACT, and (d) circular-view PACT. 
region. Among various types of NPs, gold NPs have been extensively used for PA contrast enhancement due to their strong NIR absorption, good biocompatibility, and durable structure. So far, four types of gold NPs have been used in PAT. They are nanoshells, nanocages, hollow nanospheres, and nanostars. Gold nanoshells are inorganic, optically tunable NPs consisting of a dielectric core (silica) surrounded by a thin-metallic layer (gold). Their optical resonance can vary precisely over a wide spectral range by adjusting the core size and thickness of the gold shell. ${ }^{40}$ Nanocages and hollow nanospheres are both hollow nanostructures, which exhibit improved surface plasmon resonance absorption over core-shell structures. ${ }^{69}$ Gold nanocages can be created through the reaction of Ag nanocubes and $\mathrm{HAuCl}_{4}$, and their name originates from the hollow and porous structures. ${ }^{70}$ Hollow gold nanospheres have a highly uniform structure and exhibit similar optical absorption properties as nanocages. ${ }^{69,71}$ Gold nanostars contain multiple sharp tips, which create a "lightning rod" effect to further enhance the local surface plasmon effort. ${ }^{72}$

For in vivo imaging, gold NPs are commonly coated with polyethylene glycol (PEG) to suppress the immunogenic response and subsequently improve the biostability. ${ }^{73}$ The feasibility of using PEG-coated nanoshells to enhance PAT was first demonstrated by Wang et al. ${ }^{40}$ in rat brain imaging. The nanoshells used in the experiment had a 10 to 12 -nm thick gold shell with peak absorption at $800 \pm 5 \mathrm{~nm}$. The experiment was performed on a circular-view PACT system with a 2-mm-diameter nonfocused ultrasonic transducer scanning around the rat's head. A tunable dye laser with an 800-nm emission and a $10-\mathrm{Hz}$ pulse repetition rate was used as the excitation source. Due to the slow mechanical scanning, acquiring each slice took $24 \mathrm{~min}$. After three sequential nanoshells injections through the tail vein, the absorption of blood was increased by $\sim 63 \%$ [Figs. 3(a) and 3(b)]. Similar setups have also been used to image contrast enhancements from gold nanocages ${ }^{74}$ and hollow gold nanospheres. ${ }^{41}$

Recently, an array-based circular-view PACT system has been used to image contrast enhancement from gold nanostars [Figs. 3(c) and 3(d)]. ${ }^{72}$ The system had 512 transducer elements evenly distributed on a $5-\mathrm{cm}$ diameter circle. Each element was arc-shaped with a $1.9-\mathrm{cm}$ radius of curvature along elevation direction. This design ensured rejection of out-of-plane signals. ${ }^{75}$ The array system eliminates mechanical scanning and thus significantly improves the imaging speed-a 2-D image can be captured in $0.625 \mathrm{~s}$. To match the absorption peak of nanostars, a Ti-Sapphire laser with 800 -nm output was used as the excitation source. The maximum light intensity on mouse scalp was approximately $15 \mathrm{~mJ} / \mathrm{cm}^{2}$, which is below the American National Standard Institute (ANSI) safety limit of $30 \mathrm{~mJ} / \mathrm{cm}$. The high imaging speed allows for monitoring of dynamic changes in PA amplitude. As can be seen in Fig. 3(d), the PA amplitude rose almost instantly after tail vein injection of nanostars and then it increased gradually before reaching the maximum at around half an hour. The PA signal then gradually decreased, indicating the clearance of nanostars the reticuloendothelial system (RES).

\subsection{Tumor Targeting}

In addition to vascular contrast enhancement, NPs have also been used in tumor targeting. ${ }^{76,77}$ The targeting mechanism can be either passive or active. Passive targeting is based on the enhanced permeability and retention effect (EPR effect), ${ }^{78,79}$
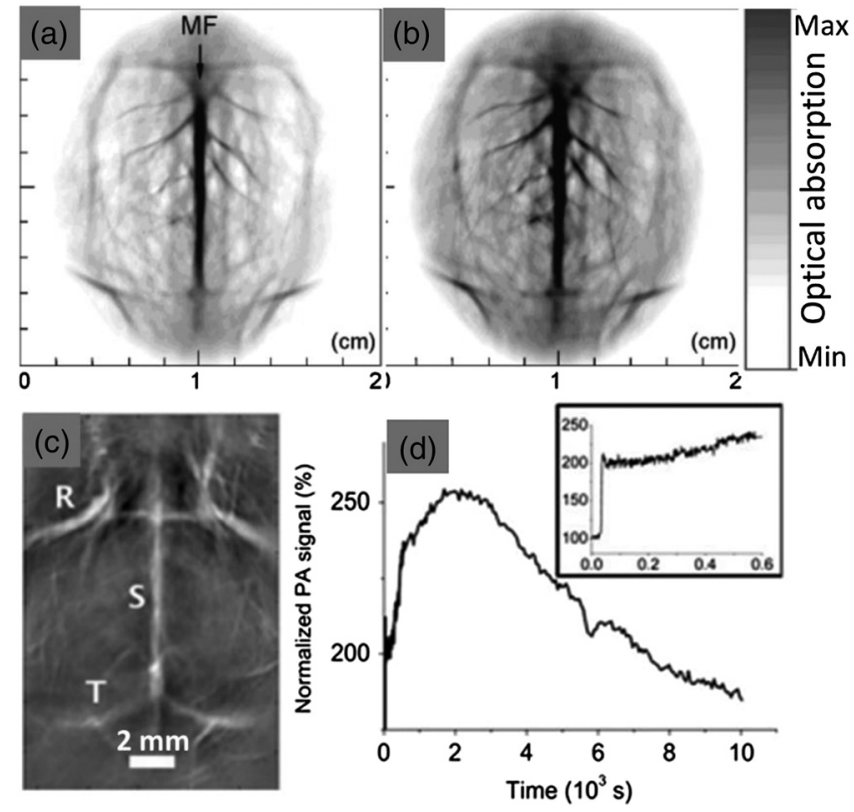

Fig. 3 Noninvasive photoacoustic (PA) neuroimaging employing gold nanoparticles (NPs). (a) and (d) PA images acquired through an intact rat scalp before and 20 min after tail vein injection of gold nanoshells, respectively. The experiment was performed on a single-elementbased circular-view PACT system. (c) PA image acquired through an intact mouse scalp after nanostar injection. R: rostral rhinal vein. $\mathrm{S}$ : sagittal sinus. T: transverse sinus. (d) PA intensity (normalized to the first frame) of sagittal sinus calculated from each frame. Inset, normalized PA intensity monitored for the initial $600 \mathrm{~s}$. The experiment was performed on an array-based circular-view PACT system. Reproduced with permission from Refs. 40 and 72.

which allows NPs to accumulate at a higher concentration in tumors than in healthy tissues. ${ }^{80}$ The EPR effect relies on two mechanisms: first, the porous vascular structures in tumors facilitate the perfusion of NPs; second, once NPs are in a tumor, they tend to stay for a prolonged period of time because of poor lymphatic drainage in the tumor, leading to a higher NP concentration.

Passive brain tumor targeting was recently demonstrated in PA neuroimaging using organic PDI NPs. ${ }^{23}$ In this study, PDI NPs had an average size of $50 \mathrm{~nm}$ and exhibited strong absorption at $700 \mathrm{~nm}$, which enabled deep-brain tumor imaging. The experiment was performed using a commercial linear-view PACT system (Vevo LARZ, Visualsonics) with a LZ-250 linear array (13-24 MHz central frequency) and a tunable OPO laser system $(680-970 \mathrm{~nm})$ with a $20 \mathrm{~Hz}$ pulse repetition rate. When all 256 elements were used for imaging, the LARZ system had a frame rate of $5 \mathrm{~Hz}$. The experiment was performed on mice with orthotopic glioblastoma tumors. Before the PA experiment, the authors first scanned the mouse with an MRI to confirm tumor size and location. They then injected $250 \mu \mathrm{L}$ of $250 \mathrm{nM}$ PDI NPs through the tail vein and imaged the animal with the LARZ system at 0 hour, 2 hours, 1 day, and 2 days post injection. Figure 4 shows coronal-view images of the mouse brain. The red dashed line indicates the skull, while the blue dashed circle indicates the tumor region. An obvious increase of PA amplitude at the tumor site can be observed starting from day 1. The depth of the tumor was estimated to be $4 \mathrm{~mm}$, which agreed with the MRI image. The tumor contrast continued to increase in day 2 , indicating enhanced tumor penetration 

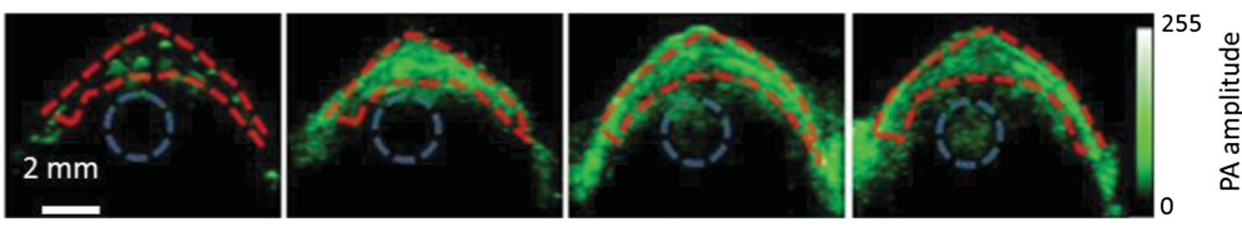

Fig. 4 Coronal-view PA images demonstrating the effect of passive tumor targeting with PDI NPs. Images were acquired after tail vein injection of $250 \mu \mathrm{L}$ of $250 \mathrm{nM}$ PDI NPs in a mouse with an orthotopic glioblastoma tumor. Adapted with permission from Ref. 23.

of NPs with time, while the skull contrast started to decrease, indicating the clearance of NPs in the circulatory system. ${ }^{23}$

Despite encouraging results, passive targeting has its inherent limitations. The cells of RES will remove most of the intravascular injected NPs, ${ }^{81}$ and the spleen, liver, lung, bone marrow, and kidney would also uptake NPs. Thus, only a small fraction of administered NPs can reach the tumor. ${ }^{82,83}$ To increase the circulation time, the size of the NPs needs to be less than $100 \mathrm{~nm}$, and the surface biocompatibility needs to be carefully designed.

Another targeting mechanism is active targeting, which uses the receptors that are highly expressed on the surface of tumor cells, such as the epidermal growth factor receptors (EGFR) and integrin. ${ }^{84}$ When NPs are decorated with ligands that can specifically bind to these receptors, the concentration of NPs in the tumor will increase. Because of the large surface area of NPs, many ligands, such as proteins and peptides, can be coated on NPs. ${ }^{85}$ Active targeting complements passive targeting with the EPR effect to provide prolonged and even targeted accumulation of NPs in tumors.

A preliminary demonstration of activate tumor targeting was carried out using organic Blue NPs, which were made of a PAA matrix with covalently linked CB dye molecules and were conjugated with $\mathrm{F} 3$ peptide. ${ }^{22}$ The $\mathrm{F} 3$ peptide binds to tumor endothelial cells while the $\mathrm{CB}$ dye enhances optical absorption at $590 \mathrm{~nm}$. The study was performed on rats with 9L glioma tumors in their brains. Animals were euthanized several days after a tail vein injection of NPs to avoid untargeted accumulation. Subsequently, the brains were excised for ex vivo PAT. The experiment was performed on a circular-view PACT system with a single, cylindrically focused $9 \mathrm{MHz}$ transducer. A dye laser (ND6000, Continuum) with a $10 \mathrm{~Hz}$ pulse repetition rate and an output at $590 \mathrm{~nm}$ (the absorption peak for NPs) was used as the excitation source. The incident light intensity
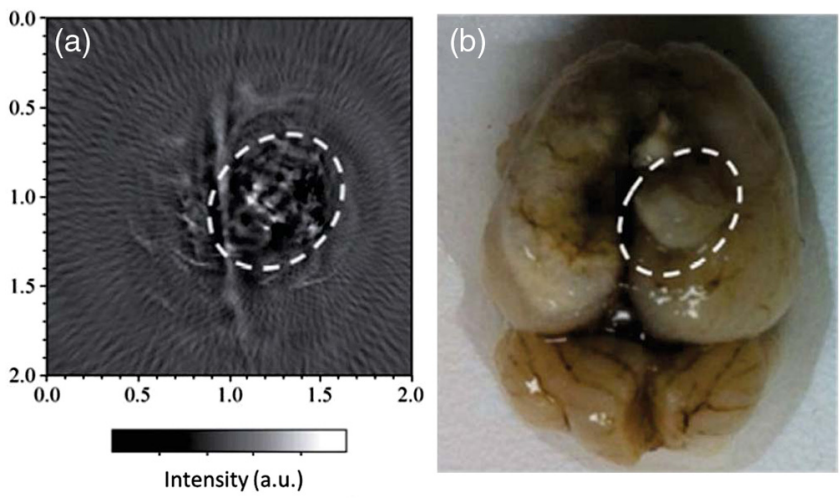

Fig. 5 (a) PA image of an excised rat brain containing a tumor treated with nanoshells. Field of view: $2 \mathrm{~cm} \times 2 \mathrm{~cm}$. (b) Photograph of the brain in (a). Adapted with permission from Ref. 22. was $3 \mathrm{~mJ} / \mathrm{cm}^{2}$, which was well below the American National Standard Institute limit of $20 \mathrm{~mJ} / \mathrm{cm}^{2}$. Because of NPs' strong optical absorption and targeting properties, the brain tumor could be clearly delineated in the PA image [Fig. 5(a)], even when the dose of NPs $(125 \mathrm{mg} / \mathrm{kg}$ of rat mass) was at a level indistinguishable by the naked eye in the photograph [Fig. 5(b)]. This study demonstrates the potential of intraoperative tumor delineation using PAT.

\subsection{Multimodal Imaging}

Multimodal imaging allows for a more comprehensive characterization of a brain in its healthy and diseased states. ${ }^{86}$ NPs are one of the most attractive multimodal contrast agents because their multicomponent nature offers many possibilities to incorporate various imaging probes. Because the same NPs can be visualized in different imaging modalities, they allow precise coregistration of multimodal images and corresponding tissue information.

A notable multimodal probe used in PA neuroimaging is trimodal MRI-PAT-Raman imaging (MPR) NPs ${ }^{87}$ MPR NPs consisted of a 60-nm gold core covered with a Raman active layer, which was further coated with silica and $\mathrm{Gd}^{3+}$, resulting in a gold-silica-based NP with both Raman and magnetic contrasts. MPR NP performances were measured on glioblastoma-bearing mice with contrasts injected through the tail vein. Imaging experiments were performed several hours after injection to let MPR NPs gradually accumulate in the tumors through the passive EPR effect. PA images were acquired by an ARPAM system. However, unlike typical AR-PAM systems with $\mathrm{kHz}$ repetition rate lasers, their system employed a $10-\mathrm{Hz}$ Nd:YAG pulsed laser (Nd:YAG laser), whose 532-nm output was coupled into a ring fiber optical bundle. A 5-MHz focused transducer was placed at the center of the fiber bundle for signal acquisition. The spatial resolution of the system was 0.6 and $0.38 \mathrm{~mm}$ along lateral and axial resolutions, respectively. Figure 6 indicates that MPR NPs can be clearly visualized in all three modalities. The MRI anatomical image allows for the precise localization of tumor position, PAT provides highresolution 3-D images of the tumor, and Raman enables surface imaging of tumor margins with high sensitivity and high specificity. ${ }^{87}$

There are many other multimodal NPs that show great potential for PA neuroimaging. For instance, because of strong optical absorption, most NPs also allow PT tumor therapy and PAT. ${ }^{88,89}$ PAT provides high-resolution images of NP distribution and tumor vasculature and offers real-time monitoring of temperature rise during PT therapy. ${ }^{72,90}$ Single-walled carbon nanotubes have enabled multimodal thermoacoustic tomography and PAT, due to their intrinsic absorption at both optical and radio frequencies. ${ }^{91}$ QDs have been used in multimodal PAT and 
MRI

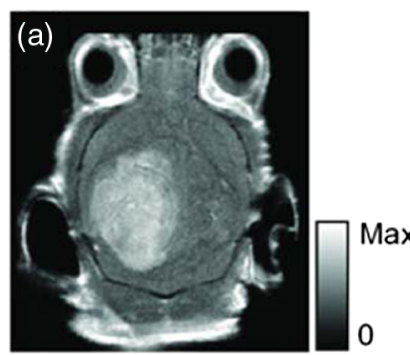

MRI+PAT

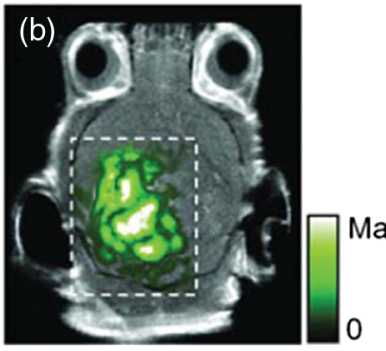

MRI+Raman

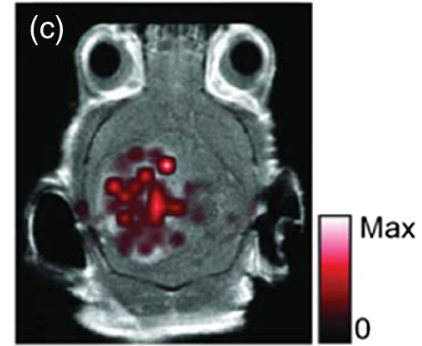

Fig. 6 MPR multimodal images of a mouse brain. (a) Magnetic resonance imaging (MRI) image, (b) PA image (green) superimposed on MRI image, and (c) Raman (red) superimposed on MRI image. Reproduced with permission from Ref. 87.

fluorescence imaging, due to their broad excitation spectra (400 to $700 \mathrm{~nm})$ and narrow emission spectra $(30$ to $80 \mathrm{~nm}) .^{44}$

\section{Conclusion}

Because of their unique properties and advantages, NPs have been increasingly used in PA neuroimaging. This review provided an overview of NPs used in PAT and highlighted their applications in PA neuroimaging. However, PAT NPs only cover a very small variety of all NPs that have been developed so far. The major impediment is that many NPs, such as the clinically approved iron oxide NPs, do not exhibit strong absorption in the red or NIR region, which is essential for deep-brain PAT. Modification of surface coating or encapsulating with light absorption materials is needed to improve the PA contrast of those NPs. There are also many other exciting applications that have not been demonstrated in PA neuroimaging. For instance, because the plasmonic resonance frequencies depend on the dielectric properties of the media, metallic NPs can potentially be used to sense electrostatic fields induced by neural firing. ${ }^{92}$ This would offer a significant advantage over dye-based action potential imaging because most voltage-sensitive dyes operate in the visible region. ${ }^{93}$ NPs have also been used to measure brain glucose levels, which provide insight on cerebral metabolism. ${ }^{94}$ This technique can potentially replace brain fludeoxyglucose positron emission tomography, which contains ionizing radiation. PA guidance of brain therapy using NPs loaded with drugs is also a very promising application. ${ }^{95}$ Therapeutic drugs can be encapsulated in an NP to be delivered and attached to the brain tumor site. Upon external excitation, the drug can be released on site, minimizing damage to healthy brain tissue. PAT can be used to monitor the process of drug release and provide real-time feedback on tissue function.

However, while preclinical imaging results are encouraging, there is still a long way to go before NPs can be used for clinical PA neuroimaging. On the NP side, the BBB crossing efficiency and biosafety need further investigation. For studies discussed in Sec. 4.2, ${ }^{23}$ NPs reached the brain tumor based on diffusion through disrupted $\mathrm{BBB}$ (due to the presence of tumor). However, the passive diffusion has a very low-crossing efficiency and only works on certain brain disorders, such as the traumatic brain injury and malignant brain tumors. Recent progresses on receptor-mediated transcytosis have shown great promise to address this issue. ${ }^{96}$ They are based on the fact that brain cells require a constant supply of nutrition, e.g., iron and glucose, to maintain their function. These nutrients and carrier proteins are able to bind with receptors expressed on brain endothelial cells and cross the BBB through receptor-mediated transcytosis. When NPs are coated with certain molecules, such as transferrin and insulin, they can be coupled to the corresponding receptors on the brain endothelial cells and be escorted through BBB. Alternatively, instead of systemic delivery through the tail vein, transnasal delivery can be used on lipid-soluble small molecules to bypass the BBB. ${ }^{97}$ As for biosafety, there is still little information on the potential toxicity of NPs to human health. As can be seen from Sec. 2, NPs can be composited from a wide variety of materials, whose bioeffects may greatly vary. PEG coating is a common approach to prevent potential toxicity of NPs. ${ }^{98}$ However, PEG-coated NPs may still be trapped in the liver and spleen for a prolonged period and their long-term effect is still unclear. BBB-penetrating NPs may also affect the central nervous system. Their neurotoxicity is also a very poorly understood area. ${ }^{99}$

On the PA side, several technical improvements are also needed. Current PAT systems are relatively bulky and can only operate on an optical table to image anesthetized animals. There is a need for a miniaturized PAT system that can be mounted on the animal head to image freely moving animals (similar to the widely used Inscopix cameras). This may open a new avenue for monitoring behavior-related neuro and hemodynamic activities using PAT. Recently, a promising progress in this direction was reported. ${ }^{21}$ Another emerging need for PAT is to minimize the acoustic distortion from skull. Even for mice imaging, the skull and bone cavities may severely degrade deep brain imaging quality. To address this issue, the bone profile needs to be mapped through another modality, such as X-ray computed tomography or ultrasound computed tomography, and be incorporated into PAT reconstruction. ${ }^{100}$

Nevertheless, with increasing effort in NP research and PA imaging, great progresses have been shown to overcome the aforementioned limitations. ${ }^{101-103}$ In the next few years, we expect to see a wider adoption of NPs in PA neuroimaging to improve our understanding of the brain and to advance the treatment of brain diseases.

\section{Acknowledgments}

This work was sponsored by University at Buffalo's Start Up fund.

\section{References}

1. L. V. Wang and S. Hu, "Photoacoustic tomography: in vivo imaging from organelles to organs," Science 335(6075), 1458-1462 (2012).

2. M. Xu and L. V. Wang, "Photoacoustic imaging in biomedicine," Rev. Sci. Instrum. 77(4), 041101 (2006). 
3. H. Xu et al., "Nanoscale optical probes for cellular imaging," Chem. Soc. Rev. 43(8), 2650-2661 (2014).

4. X. Wang et al., "Noninvasive photoacoustic angiography of animal brains in vivo with near-infrared light and an optical contrast agent," Opt. Lett. 29(7), 730-732 (2004).

5. D. Razansky et al., "Multispectral opto-acoustic tomography of deepseated fluorescent proteins in vivo," Nat. Photonics 3(7), 412-417 (2009).

6. G. S. Filonov et al., "Deep-tissue photoacoustic tomography of a genetically encoded near-infrared fluorescent probe," Angew. Chem. 124(6), 1477-1480 (2012).

7. J. Yao and L. V. Wang, "Photoacoustic brain imaging: from microscopic to macroscopic scales," Neurophotonics 1(1), 011003 (2014).

8. J. Xia and L. V. Wang, "Photoacoustic tomography of the brain," in Optical Methods and Instrumentation in Brain Imaging and Therapy, pp. 137-156, Springer, New York (2013).

9. S. Hu and L. V. Wang, "Neurovascular photoacoustic tomography," Front. Neuroenerg. 3, 1-7 (2010).

10. H. Chen, Z. Yuan, and C. Wu, "Nanoparticle probes for structural and functional photoacoustic molecular tomography," BioMed Res. Int. 2015, 1-11 (2015).

11. C. Kim, C. Favazza, and L. V. Wang, "In vivo photoacoustic tomography of chemicals: high-resolution functional and molecular optical imaging at new depths," Chem. Rev. 110(5), 2756-2782 (2010).

12. L. Nie and X. Chen, "Structural and functional photoacoustic molecular tomography aided by emerging contrast agents," Chem. Soc. Rev. 43(20), 7132-7170 (2014).

13. F. Alexis et al., "Factors affecting the clearance and biodistribution of polymeric nanoparticles," Mol. Pharm. 5(4), 505-515 (2008).

14. J. L. West and N. J. Halas, "Engineered nanomaterials for biophotonics applications: improving sensing, imaging, and therapeutics," Аппи. Rev. Biomed. Eng. 5(1), 285-292 (2003).

15. M. Holzinger, A. Le Goff, and S. Cosnier, "Nanomaterials for biosensing applications: a review," Front. Chem. 3, 1-10 (2014).

16. J. F. Lovell et al., "Porphysome nanovesicles generated by porphyrin bilayers for use as multimodal biophotonic contrast agents," Nat. Mater. 10(4), 324-332 (2011).

17. A. Hannah et al., "Indocyanine green-loaded photoacoustic nanodroplets: dual contrast nanoconstructs for enhanced photoacoustic and ultrasound imaging," ACS Nano 8(1), 250-259 (2013).

18. W. J. Akers et al., "Noninvasive photoacoustic and fluorescence sentinel lymph node identification using dye-loaded perfluorocarbon nanoparticles," ACS Nano 5(1), 173-182 (2010).

19. Y. Li et al., "A dual-targeting nanocarrier based on poly (amidoamine) dendrimers conjugated with transferrin and tamoxifen for treating brain gliomas," Biomaterials 33(15), 3899-3908 (2012).

20. H. Xin et al., "Anti-glioblastoma efficacy and safety of paclitaxel-loading Angiopep-conjugated dual targeting PEG-PCL nanoparticles," Biomaterials 33(32), 8167-8176 (2012).

21. J. Tang et al., "Noninvasive high-speed photoacoustic tomography of cerebral hemodynamics in awake-moving rats," J. Cerebral Blood Flow Metab. 35(8), 1224-1232 (2015).

22. A. Ray et al., "Targeted blue nanoparticles as photoacoustic contrast agent for brain tumor delineation," Nano Res. 4(11), 1163-1173 (2011).

23. Q. Fan et al., "Perylene-diimide-based nanoparticles as highly efficient photoacoustic agents for deep brain tumor imaging in living mice," Adv. Mater. 27(5), 843-847 (2015).

24. O. D. Kripfgans et al., "Acoustic droplet vaporization for therapeutic and diagnostic applications," Ultrasound Med. Biol. 26(7), 1177-1189 (2000).

25. T. Giesecke and K. Hynynen, "Ultrasound-mediated cavitation thresholds of liquid perfluorocarbon droplets in vitro," Ultrasound Med. Biol. 29(9), 1359-1365 (2003).

26. P. S. Sheeran and P. A. Dayton, "Phase-change contrast agents for imaging and therapy," Curr. Pharm. Des. 18(15), 2152-2165 (2012).

27. N. Reznik et al., "Optical studies of vaporization and stability of fluorescently labelled perfluorocarbon droplets," Phys. Med. Biol. 57(21), 7205 (2012).

28. K. Pu et al., "Semiconducting polymer nanoparticles as photoacoustic molecular imaging probes in living mice," Nat. Nanotechnol. 9(3), 233-239 (2014).
29. J. Chen et al., "Gold nanocages: bioconjugation and their potential use as optical imaging contrast agents," Nano Lett. 5(3), 473-477 (2005).

30. W. C. W. Chan et al., "Luminescent quantum dots for multiplexed biological detection and imaging," Curr. Opin. Biotechnol. 13(1), 40-46 (2002).

31. X. Gao et al., "In vivo molecular and cellular imaging with quantum dots," Curr. Opin. Biotechnol. 16(1), 63-72 (2005).

32. X. Feng, F. Gao, and Y. Zheng, "Thermally modulated photoacoustic imaging with super-paramagnetic iron oxide nanoparticles," Opt. Lett. 39(12), 3414-3417 (2014).

33. Y. Jin et al., "Multifunctional nanoparticles as coupled contrast agents," Nat. Commun. 1, 41 (2010).

34. A. De La Zerda et al., "Carbon nanotubes as photoacoustic molecular imaging agents in living mice," Nat. Nanotechnol. 3(9), 557-562 (2008).

35. S. Zanganeh et al., "Photoacoustic imaging enhanced by indocyanine green-conjugated single-wall carbon nanotubes," J. Biomed. Opt. 18(9), 096006 (2013).

36. P. K. Avti et al., "Detection, mapping, and quantification of single walled carbon nanotubes in histological specimens with photoacoustic microscopy," PLoS One 7(4), e35064 (2012).

37. V. P. Nguyen et al., "Enhancement of high-resolution photoacoustic imaging with indocyanine green-conjugated carbon nanotubes," Jpn. J. Appl. Phys. 54(7S1), $07 \mathrm{HF} 04$ (2015).

38. S. K. Maji et al., "Upconversion nanoparticles as a contrast agent for photoacoustic imaging in live mice," Adv. Mater. 26(32), 5633-5638 (2014).

39. J. Chen et al., "Gold nanocages: engineering their structure for biomedical applications," Adv. Mater. 17(18), 2255-2261 (2005).

40. Y. Wang et al., "Photoacoustic tomography of a nanoshell contrast agent in the in vivo rat brain," Nano Lett. 4(9), 1689-1692 (2004).

41. W. Lu et al., "Photoacoustic imaging of living mouse brain vasculature using hollow gold nanospheres," Biomaterials 31(9), 2617-2626 (2010).

42. G. Xu et al., "Theranostic quantum dots for crossing blood-brain barrier in vitro and providing therapy of HIV-associated encephalopathy," Front. Pharmacol. 4, 1-8 (2013).

43. S. Hanada et al., "Cell-based in vitro blood-brain barrier model can rapidly evaluate nanoparticles' brain permeability in association with particle size and surface modification," Int. J. Mol. Sci. 15(2), 1812-1825 (2014).

44. E. V. Shashkov et al., "Quantum dots as multimodal photoacoustic and photothermal contrast agents," Nano Lett. 8(11), 3953-3958 (2008).

45. R. Weissleder et al., "Experimental lymph node metastases: enhanced detection with MR lymphography," Radiology 171(3), 835-839 (1989).

46. R. Alwi et al., "Silica-coated super paramagnetic iron oxide nanoparticles (SPION) as biocompatible contrast agent in biomedical photoacoustics," Biomed. Opt. Express 3(10), 2500-2509 (2012).

47. W. S. Enochs et al., "Improved delineation of human brain tumors on MR images using a long-circulating, superparamagnetic iron oxide agent," J. Magn. Reson. Imaging 9(2), 228-232 (1999).

48. A. S. Hannah et al., "Photoacoustic and ultrasound imaging using dual contrast perfluorocarbon nanodroplets triggered by laser pulses at 1064 nm," Biomed. Opt. Express 5(9), 3042-3052 (2014).

49. L. V. Wang, "Ultrasound-mediated biophotonic imaging: a review of acousto-optical tomography and photo-acoustic tomography," Disease Markers 19(2-3), 123-138 (2004).

50. J. Xia and L. V. Wang, "Small-animal whole-body photoacoustic tomography: a review," IEEE Trans. Biomed. Eng. 61(5), 13801389 (2014).

51. J. Yao et al., "High-speed label-free functional photoacoustic microscopy of mouse brain in action," Nat. Methods 12(5), 407-410 (2015).

52. L. Wang, K. Maslov, and L. V. Wang, "Single-cell label-free photoacoustic flowoxigraphy in vivo," Proc. Natl. Acad. Sci. 110(15), 5759-5764 (2013).

53. X. Yang et al., "Photoacoustic tomography of small animal brain with a curved array transducer," J. Biomed. Opt. 14(5), 054007 (2009).

54. L. Song, K. Maslov, and L. V. Wang, "Multifocal optical-resolution photoacoustic microscopy in vivo," Opt. Lett. 36(7), 1236-1238 (2011). 
55. G. Li, K. I. Maslov, and L. V. Wang, "Reflection-mode multifocal optical-resolution photoacoustic microscopy," J. Biomed. Opt. 18(3), 030501 (2013).

56. J. Xia et al., "Wide-field two-dimensional multifocal optical-resolution photoacoustic-computed microscopy," Opt. Lett. 38(24), 5236-5239 (2013).

57. S. Gottschalk et al., "Noninvasive real-time visualization of multiple cerebral hemodynamic parameters in whole mouse brains using fivedimensional optoacoustic tomography," J. Cerebral Blood Flow Metab. 35(4), 531-535 (2015).

58. X. Wang et al., "Three-dimensional laser-induced photoacoustic tomography of mouse brain with the skin and skull intact," Opt. Lett. 28(19), 1739-1741 (2003).

59. J. Laufer et al., "Three-dimensional noninvasive imaging of the vasculature in the mouse brain using a high resolution photoacoustic scanner," Appl. Opt. 48(10), D299-D306 (2009).

60. L. Xiang et al., "4-D photoacoustic tomography," Sci. Rep. 3, 1-8 (2013).

61. Z. Guo, S. Hu, and L. V. Wang, "Calibration-free absolute quantification of optical absorption coefficients using acoustic spectra in 3D photoacoustic microscopy of biological tissue," Opt. Lett. 35(12), 2067-2069 (2010).

62. M. Xu and L. V. Wang, "Universal back-projection algorithm for photoacoustic computed tomography," Proc. SPIE 5697, 251-254 (2005).

63. D. Finch, M. Haltmeier, and Rakesh, "Inversion of spherical means and the wave equation in even dimensions," SIAM J. Appl. Math. 68(2), 392-412 (2007).

64. K. Wang and M. A. Anastasio, "A simple Fourier transform-based reconstruction formula for photoacoustic computed tomography with a circular or spherical measurement geometry," Phys. Med. Biol. 57(23), N493 (2012).

65. J. Xia et al., "Three-dimensional photoacoustic tomography based on the focal-line concept," J. Biomed. Opt. 16(9), 090505 (2011).

66. J. Gateau et al., "Single-side access, isotropic resolution, and multispectral three-dimensional photoacoustic imaging with rotate-translate scanning of ultrasonic detector array," J. Biomed. Opt. 20(5), 056004 (2015).

67. X. Wang et al., "Noninvasive laser-induced photoacoustic tomography for structural and functional in vivo imaging of the brain," Nat. Biotechnol. 21(7), 803-806 (2003).

68. M. Nasiriavanaki et al., "High-resolution photoacoustic tomography of resting-state functional connectivity in the mouse brain," Proc. Natl. Acad. Sci. 111(1), 21-26 (2014).

69. A. M. Schwartzberg et al., "Synthesis, characterization, and tunable optical properties of hollow gold nanospheres," J. Phys. Chem. B 110(40), 19935-19944 (2006).

70. W. Li et al., "Gold nanocages as contrast agents for photoacoustic imaging," Contrast Media Mol. Imaging 6(5), 370-377 (2011).

71. M. P. Melancon et al., "In vitro and in vivo targeting of hollow gold nanoshells directed at epidermal growth factor receptor for photothermal ablation therapy," Mol. Cancer Ther. 7(6), 1730-1739 (2008).

72. H. Yuan et al., "Plasmonics-enhanced and optically modulated delivery of gold nanostars into brain tumor," Nanoscale 6(8), 4078-4082 (2014).

73. N. E. Martin and M. Modi, "Pegylation: a novel process for modifying pharmacokinetics," Clin. Pharmacokinet. 40(7), 539-551 (2001).

74. X. Yang et al., "Photoacoustic tomography of a rat cerebral cortex in vivo with Au nanocages as an optical contrast agent," Nano Lett. 7(12), 3798-3802 (2007).

75. J. Gamelin et al., "A real-time photoacoustic tomography system for small animals," Opt. Express 17(13), 10489-10498 (2009).

76. G. Nie et al., "Hydrogel nanoparticles with covalently linked Coomassie blue for brain tumor delineation visible to the surgeon," Small 8(6), 884-891 (2012).

77. S. M. Moghimi, A. C. Hunter, and J. C. Murray, "Long-circulating and target-specific nanoparticles: theory to practice," Pharmacol. Rev. 53(2), 283-318 (2001).

78. H. Maeda et al., "Vascular permeability enhancement in solid tumor: various factors, mechanisms involved and its implications," Int. Immunopharmacol. 3(3), 319-328 (2003).
79. H. F. Dvorak et al., "Identification and characterization of the blood vessels of solid tumors that are leaky to circulating macromolecules," Am. J. Pathol. 133(1), 95 (1988).

80. Y. Matsumura and H. Maeda, "A new concept for macromolecular therapeutics in cancer chemotherapy: mechanism of tumoritropic accumulation of proteins and the antitumor agent smancs," Cancer Res. 46(12 Part 1), 6387-6392 (1986).

81. M. A. Dobrovolskaia and S. E. McNeil, "Immunological properties of engineered nanomaterials," Nat. Nanotechnol. 3(8), 469-478 (2007).

82. N. Khlebtsov and L. Dykman, "Biodistribution and toxicity of engineered gold nanoparticles: a review of in vitro and in vivo studies," Chem. Soc. Rev. 40(3), 1647-1671 (2011).

83. H. Yan et al., "Two-order targeted brain tumor imaging by using an optical/paramagnetic nanoprobe across the blood brain barrier," ACS nano 6(1), 410-420 (2011).

84. J. S. Desgrosellier and D. A. Cheresh, "Integrins in cancer: biological implications and therapeutic opportunities," Nat. Rev. Cancer 10(1), 9-22 (2010).

85. M. Qin, H. Zong, and R. Kopelman, "Click conjugation of peptide to hydrogel nanoparticles for tumor-targeted drug delivery," Biomacromolecules 15(10), 3728-3734 (2014).

86. J. Mansik and K. Chulhong, "Multimodal photoacoustic tomography," IEEE Trans. Multimedia 15(5), 975-982 (2013).

87. M. F. Kircher et al., "A brain tumor molecular imaging strategy using a new triple-modality MRI-photoacoustic-Raman nanoparticle," Nat. Med. 18(5), 829-834 (2012).

88. J. W. Kim et al., "Golden carbon nanotubes as multimodal photoacoustic and photothermal high-contrast molecular agents," Nat. Nanotechnol. 4(10), 688-694 (2009).

89. H. W. Yang et al., "Magnetic gold-nanorod/PNIPAAmMA nanoparticles for dual magnetic resonance and photoacoustic imaging and targeted photothermal therapy," Biomaterials 34(22), 5651-5660 (2013).

90. J. Shah et al., "Photoacoustic imaging and temperature measurement for photothermal cancer therapy," J. Biomed. Opt. 13(3), 034024 (2008).

91. M. Pramanik et al., "Single-walled carbon nanotubes as a multimodalthermoacoustic and photoacoustic-contrast agent," J. Biomed. Opt. 14(3), 034018 (2009).

92. J. Zhang, T. Atay, and A. V. Nurmikko, "Optical detection of brain cell activity using plasmonic gold nanoparticles," Nano Lett. 9(2), 519-524 (2009).

93. S. Chemla and F. Chavane, "Voltage-sensitive dye imaging: technique review and models," J. Physiol. 104(1), 40-50 (2010).

94. F. F. Zhang et al., "Amperometric sensor based on ferrocene-doped silica nanoparticles as an electron transfer mediator for the determination of glucose in rat brain coupled to in vivo microdialysis," J. Electroanal. Chem. 571(2), 133-138 (2004).

95. J. Xia, C. Kim, and J. F Lovell, "Opportunities for photoacousticguided drug delivery," Curr. Drug Targets 16(6), 571-581 (2015).

96. K. K. Jain, "Nanobiotechnology-based strategies for crossing the blood-brain barrier," Nanomedicine 7(8), 1225-1233 (2012).

97. W. M. Pardridge, "Blood-brain barrier delivery," Drug Discovery Today 12(1-2), 54-61 (2007).

98. C. S. Yah, "The toxicity of gold nanoparticles in relation to their physiochemical properties," Biomed. Res. 24(3), 400-413 (2013).

99. Y. L. Hu and J. Q. Gao, "Potential neurotoxicity of nanoparticles," Int. J. Pharm. 394(1), 115-121 (2010).

100. C. Huang et al., "Full-wave iterative image reconstruction in photoacoustic tomography with acoustically inhomogeneous media," IEEE Trans. Med. Imaging 32(6), 1097-1110 (2013).

101. S. N. Tabatabaei et al., "Remote control of the permeability of the blood-brain barrier by magnetic heating of nanoparticles: a proof of concept for brain drug delivery," J. Controlled Release 206, 49-57 (2015).

102. H. Yuan et al., "Optically enhanced blood-brain-barrier crossing of plasmonic-active nanoparticles in preclinical brain tumor animal models," Proc. SPIE 8935, 89350E (2014).

103. M. Kolter et al., "Nanotoxicity of poly (n-butylcyano-acrylate) nanoparticles at the blood-brain barrier, in human whole blood and in vivo," J. Controlled Release 197, 165-179 (2015). 
Depeng Wang is currently a $\mathrm{PhD}$ student of biomedical engineering at the University at Buffalo. He received his bachelor's and master's degrees in engineering from Nanjing University of Aeronautics and Astronautics in 2012 and 2015, respectively.

Yun Wu received her PhD degree at The Ohio State University, and did postdoc training in the same university under the mentorship of Dr. Ly James Lee. She is currently an assistant professor in the Biomedical Engineering Department at the University at Buffalo and works on the development of therapeutic and diagnostic systems for the treatment and early detection of cancer and diseases. She has published more than 30 peer-reviewed journal articles in nanomedicine and bionanotechnology fields.

Jun Xia received his $\mathrm{PhD}$ at the University of Toronto and did his postdoc training at Washington University in St. Louis under the mentorship of Dr. Lihong V. Wang. Currently, he is an assistant professor in the Biomedical Engineering Department at the University at Buffalo. His research interests are the development of novel biomedical imaging techniques including photoacoustic imaging and ultrasonic imaging. He has published more than 30 peer-reviewed journal articles in photoacoustic and photothermal research. 